Journal of

Health, Medicine and Nursing

(JHMN)

\title{
INFLUENCE OF PATIENT-RELATED FACTORS ASSOCIATED ON TB OUTCOMES AMONG TB PATIENTS IN MOMBASA COUNTY
}

Godana Mamo Barako, Professor Simon Karanja and Dr. Rahma Udu

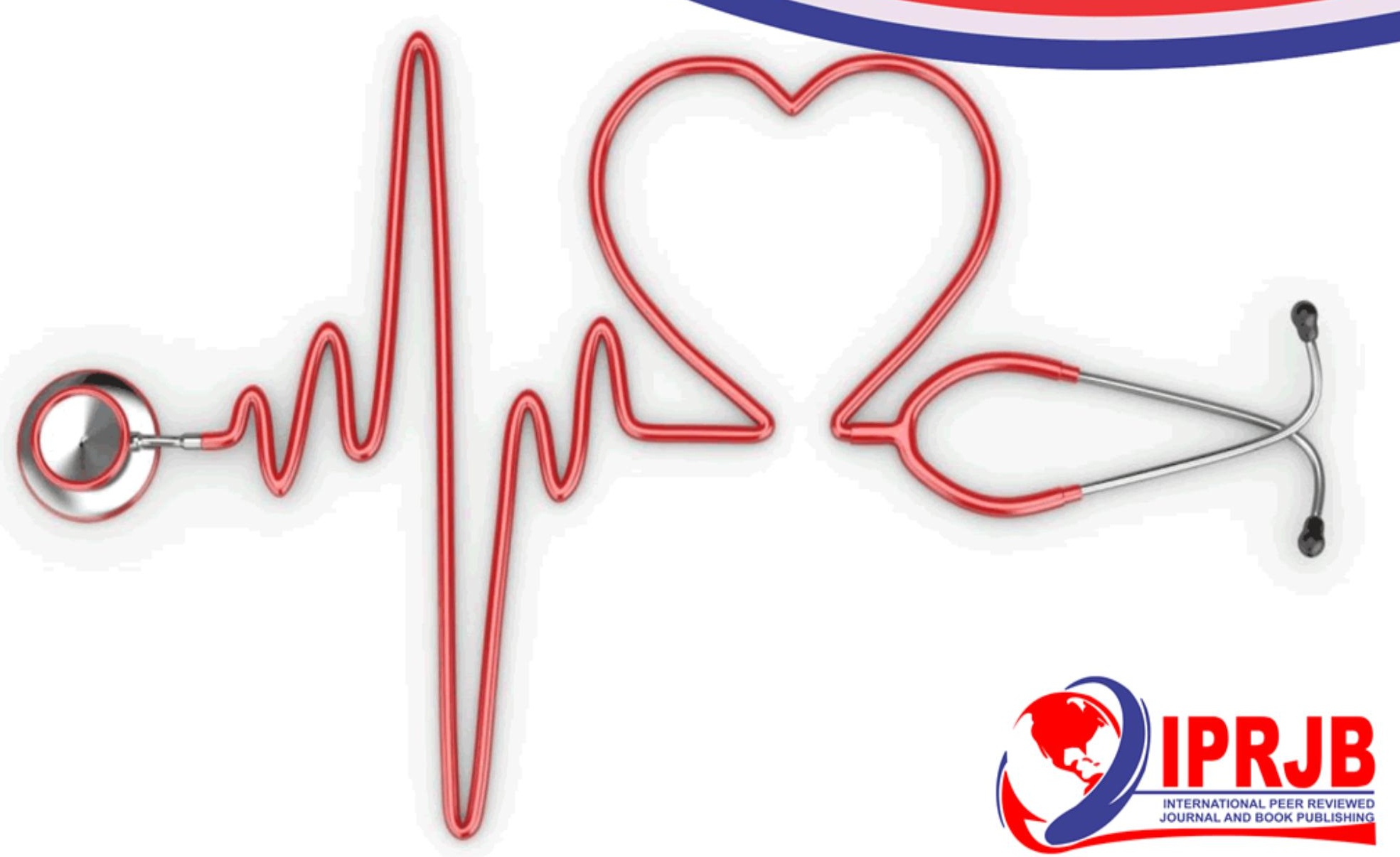


Journal of Health, Medicine and Nursing

ISSN 2520-4025 (Online)

Vol.6, Issue 3. No.4, pp 49- 65, 2021

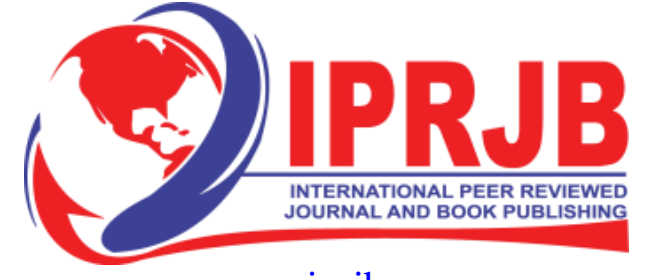

WWW.iprib.org

INFLUENCE OF PATIENT-RELATED FACTORS ASSOCIATED ON TB OUTCOMES AMONG TB PATIENTS IN MOMBASA COUNTY

\author{
${ }^{1 *}$ Godana Mamo Barako \\ Postgraduate Student, Msc Public Health: Jomo Kenyatta University of Agriculture and \\ Technology \\ Corresponding Author’s E-mail: gmamo@chskenya.org \\ ${ }^{2}$ Professor Simon Karanja \\ Lecturer: Jomo Kenyatta University of Agriculture and Technology \\ ${ }^{3}$ Dr. Rahma Udu \\ Lecturer: Jomo Kenyatta University of Agriculture and Technology
}

\begin{abstract}
Purpose: The purpose of the study was to determine patient-related factors associated with TB outcomes among TB patients in Mombasa County.

Methodology: Study was conducted in Mombasa County which is one of the 47 counties in Kenya with an urban population of 1,063,854. The study used a cross-sectional research design. The study population was the total number of notified patients with tuberculosis in one quarter in the study area (Mombasa's health care units) and this was found to be 1207 in the year 2017. It was from this population that a systematic random sample size of 292 patients were interviewed. Quantitative data were analyzed using SPSS version 20 Descriptive statistics frequency (\%), mean, and standard deviation were used to express quantitative data. In bivariate analyses, odds ratios (OR) and 95\% confidence intervals (CI) for the association between TB treatment outcome and health related factors, institutional factors and patient related factors was done using logistic regression.

Results: The results revealed that patients who are educated about health are more likely to cure of TB than patients who are not (OR 1.716, 95\% CI, 0.35 to 1.48). More so, patients who receive psychosocial support are more likely to get cured than those who don't receive psychosocial support (OR 4.08, 95\% CI, 2.00 to 8.32). The results of the study give evidence to, therefore, conclude patient related factors are critical to TB treatment outcome.

Unique contribution to theory, policy and practice: Strengthening TB adherence counselling for patients treated for the first time to improve treatment completion. Counselling service should also be enhanced for patients taking alcohol and those smoking cigarettes as both are associated with less likelihood of completing TB treatment. Implementation of TB work place program through sensitization of company managers on the importance of TB treatment support to reduce chances of loss to follow as sighted by focused group discussions. Patients centred TB services should be provided by all facilities by focusing on the needs of the patients by agreeing on the best time to pick drugs to reduce the risk of loss to follow ups.
\end{abstract}

Keywords: Patient-related factors, TB outcomes, TB patients. 
Journal of Health, Medicine and Nursing

ISSN 2520-4025 (Online)

Vol.6, Issue 3. No.4, pp 49- 65, 2021

\subsection{INTRODUCTION}

TB is caused by the bacterium Mycobacterium tuberculosis, which is an air born disease transmitted from person to person by aerosolized droplet nuclei. It typically affects the lungs (pulmonary tuberculosis) but can affect other sites as well (Varaine and Rich, 2014). The disease is spread in the air when people who are sick with pulmonary TB expel bacteria through coughing, sneezing, laughing or talking (MOPHS, 2009). Relatively small number of people infected with TB will eventually develop the active disease and the probability is much higher among the people living with HIV/AIDS (WHO, 2010).

Tuberculosis is among the top ten causes of death worldwide third among the infectious diseases overtaking even HIV (WHO, 2016). In 20128.6 million people developed the disease, 1.3 million died (WHO, 2013). World Health Organization introduced DOTS strategy in 1990 as a cost-effective way to control TB and improve health. Adoption and implementation of this strategy has substantially improved TB control globally. Kenya is one of the few countries in Africa to have achieved the WHO targets of detecting $70 \%$ of new TB cases and cure $85 \%$ of smear positive TB (WHO, 2014). Kenya is one of the 22 high burdened countries in the world ranked $13^{\text {th }}$ globally and $5^{\text {th }}$ in Africa. Between 2006 and 2010, more than 110,000 cases of TB were notified every year and about 44 per cent of these cases were HIV co-infected. The WHO estimate shows that there are at least 2000 cases of MDR-TB in 2009, of which only 7.5\% (150) cases have been identified and notified (WHO, 2011).

Tuberculosis (TB) remains a major cause of morbidity and mortality in Kenya. It affects all age groups but has its greatest toll in the most productive age group of 15 to 45 years Sitienei J, Nyambati V and Bours P (2013). The major factor responsible for the large TB disease burden is the current HIV epidemic. Other factors that have contributed to this large TB disease burden include poverty and social deprivation that has led to mushrooming of peri-urban slums and congestion. Although TB cases notified in the country has stagnated recently, a new challenge of resistant strains of TB is gradually but surely increasing and in particular MDR TB (ministry of public health and sanitation (2010). This new challenge threatens to reverse gains made in the fight against TB in Kenya. Drug Resistant TB (DRTB) is occasioned by development of resistance by the TB bacilli to first line drugs, a result of use improper treatment of patients, poor management of supply and quality of drugs, and airborne transmission of bacteria in public places, Companion handbook WHO (2014). Mombasa is one of the counties with high tuberculosis burden of 425 case notification rate against a national prevalence of 215 (National Leprosy and Lung Disease Program, 2014). More than eighty cases of multidrug resistant TB were diagnosed in the County by end of 2014 (Ministry of health, 2012). A total of twenty-six patients are currently on treatment in County.

\subsection{Statement of the problem}

Tuberculosis control has three overlapping dimensions; humanitarian, public health and economic. The public health concern emanates from proper diagnosis and treatment of TB patient to decrease disease transmission within community (Arnadottoir, 2009). TB is also responsible for direct or indirect cost to individual and society. The productive adults bear the greatest burden of $\mathrm{TB}$, once infected they are weakened and often unable to work (Laxminarayan, 2007). Tuberculosis remains a major public health problem causing losses in quality of adjusted life years (QALYS) or disability adjusted life-years (DALYs) and it ranks 7th 
Journal of Health, Medicine and Nursing

ISSN 2520-4025 (Online)

Vol.6, Issue 3. No.4, pp 49- 65, 2021

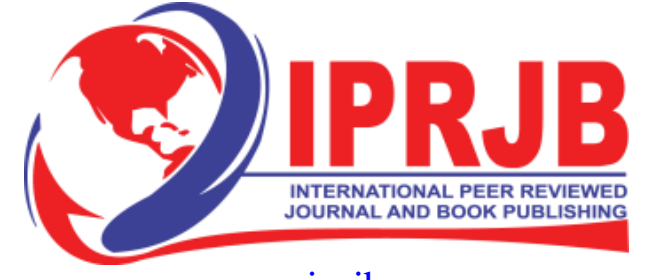

WWW.iprib.org

globally in the global life years lost ranking (Banerjee and Nasaru M, (2014). The failure to eliminate or completely reduce TB as a public health problem has been largely associated with little attention the disease attracts from the government, poor management of $\mathrm{TB}$ control programmes, high rates of poverty and population growth especially in urban areas due to migration and HIV/AIDS pandemic. Considerable progress has been made since introduction of DOTS strategy.

The world health assembly passed a resolution to end global TB epidemic with targets to reduce TB death by $95 \%$ and cut new cases by $90 \%$ between 2015 and 2035 to ensure that no family is burdened with catastrophic expenses due to TB (WHO, 2014). The WHO has recommended adoption of directly observed therapy (DOTS) as strategy to achieve high treatment completion rates in 1990. This is expected to reduce patient's loss to follow up, treatment failures, death and emergence of drug resistance associated with treatment interruptions. Studies have shown that 86-90\% of patient receiving complete treatment successfully as compared to self-administered to therapy. Treatment completion and cure rates for Mombasa County have over a long period of time remained low. This is attributable to a number of factors associated with patients, health facility and health care workers.

This project addresses the overall problem associated with patients that hinder treatment completion and also attempt to identify factors that promote TB treatment completions in Mombasa County. The county had the highest TB burden in country with $469 \mathrm{~TB}$ cases per 100,000 populations which is higher than the national prevalence of $217 / 100,000$ (NLTLD, 2013). Eleven percent of patients started on treatment in Mombasa County failed to complete full course of treatment due high rates of loss to follow up, deaths and transfers to other counties reducing treatment completion. One hundred and seventy-six TB patients died in Mombasa County in the year 2012, 6\% being children below 15 years of age in addition $49 \%$ of the patients who died were co-infected with TB/HIV mainly patients aged between 20-39 years old. Further, two hundred and twenty patients were lost to follow in the year 2012 with $23 \%$ of them in Mlaeo TB control zone. Likoni and Ganjoni contributed $16 \%$ and $14 \%$ respectively and this was attributed to substance abuse and lack of social support from close family members. One hundred and eighty-seven TB patients were also transferred to other counties across the country and could not be evaluated. Changamwe and Mlaleo TB control zones contributed more than $50 \%$ of these cases.

\subsection{Justification}

Mombasa county had the highest TB case notification rates of 469/100,000 population in the country (NLTLD, 2013). The treatment completion rates vary according to the various categories of patients with smear positive pulmonary relapse cases having the lowest at $86 \%$ and smear not done pulmonary patients having $91 \%$. This is attributable to high loss to follow ups among smear positive retreatment cases $(7 \%)$, high death rates among smear negative pulmonary cases $(5 \%)$ and transfer outs among new smear positive patients (4\%). Efforts to improve TB treatment completion rates require understanding of factors that influence patient's adherence to TB treatment. Quantitative and qualitative research can contribute to this understanding and promote treatment success rates.

\subsection{Purpose of the study}


Journal of Health, Medicine and Nursing

ISSN 2520-4025 (Online)

Vol.6, Issue 3. No.4, pp 49- 65, 2021

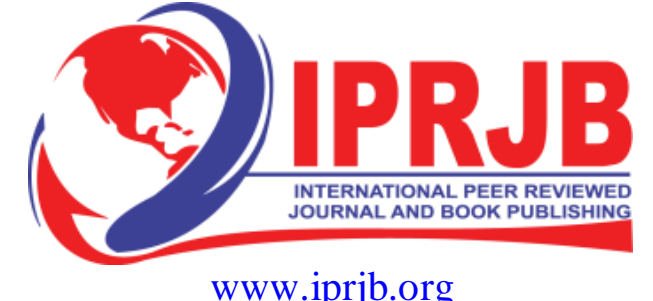

The purpose of the study was to determine patient-related factors associated with TB outcomes among TB patients in Mombasa County.

\subsection{Research Questions}

The study was guided by the following research question:

i. What are the patient-related factors associated with TB outcomes?

\subsection{LITERATURE REVIEW}

\subsection{Theoretical Review}

The most successful public health programs and initiatives are based on recognizing the health behaviour in the context they occur. As such interventions to improve health behaviour can be designed with an understanding of behaviour change models and the ability to use them skillfully. This study will be anchored by transtheoretical model which is a theory of social behaviour change. The key element in this model is stage of change and proposes that people are at different stages of readiness to adopt healthful behaviour. For successful behaviour change, the model describes the sequence of steps which an individual goes through: Precontemplation, contemplation, preparation, action and maintenance. However, people do not always follow these stages in a linear manner but often repeat certain stages depending on their level of selfefficacy and motivation.

The behaviour of patients and providers have an impact on drug adherence and patient outcomes. Patient's knowledge, attitude, perception and acceptance of TB drug adherence influence their outcome (CDC, 2003). Outcome of TB patients are influenced by types of drug regimen that they are given leading to high pill burden and long duration of treatment, patient factors, relationship between patient and providers and the system of care (Arnadottoir, 2009). Patients may drop out as symptoms subside due to clinical improvement. Geographical proximity to treatment centers, alcoholism and drug addiction may also contribute patient's loss to follow up.

In a systematic review on timing of loss to TB follow up, majority occurred after the intensive phase of treatment (Kruk, May, 2008). A study conducted in Georgia in October 2013 showed that lower household income was found to be significantly associated with poor TB treatment outcome. According to Liew et al., (2015), the commonest predictors of unfavorable outcomes were: old age, male sex, foreign citizenship, lower education, treatment in tertiary setting, smocking, previous anti-TB treatment, HIV infection and patients with extra-pulmonary TB.

\subsection{Conceptual Framework}

The conceptual framework is adopted from the socio-behavioural model advanced by (Andersen, 1995) and collated from literature review. The model framework looks at the predisposing characteristics, and enabling resources as the major factors for uptake of preconception care. Figure 1 below presents the conceptual framework for the study.

\begin{tabular}{|l|c|}
\multicolumn{1}{c}{ Independent Variable } & Dependent Variable \\
\hline Patient related factors & $\mathrm{TB}$ outcomes among TB patients \\
\hline
\end{tabular}

Figure 1: Conceptual framework 
Journal of Health, Medicine and Nursing

ISSN 2520-4025 (Online)

Vol.6, Issue 3. No.4, pp 49- 65, 2021

\section{Burden of Tuberculosis}

Tuberculosis has re-emerged as a major public health problem in the world. Roughly, it is estimated that at least a third of the word population is infected with tubercle bacillus, and around 8 million progress to have active tuberculosis disease each year, while 2 million of those die of the disease. According to WHO reports, incidences of the TB grew by $1 \%$ in the year 2003 in the world. This might seem as a low growth rate or long time ago, but recent studies have found the increase of the disease in the $19^{\text {th }}$ century having an effect in $20^{\text {th }}$ century.

Currently, about one-third of the world's population is infected with M. tuberculosis and new infections occur at a rate of one per second. However, most of these infections are asymptomatic and most of them do not cause tuberculosis disease. Tuberculosis is the second most common cause of death after HIV. In 2014, there were an estimated 13.7 million chronic active cases and in 2015 there were 8.8 million new cases and 1.34 million deaths in both developed and developing countries (WHO, 2016). China has achieved great progress, with an 80 percent decline in its TB mortality rate. Although, the distribution of tuberculosis is not uniform across the world, every nation has reported various cases of tuberculosis. In U.S, $10 \%$ of the population test positive, while Swaziland was reported to be the country with the highest estimated incidence rate of TB with 1200 cases in every 100,000 people in 2007. In 2010, India had the highest incidence with an estimated 2.0 million new cases. The reported cases in developed countries have always been attributed to the urban environment with almost infinitesimal cases in rural areas.

In Africa, 9 countries have been designated as having high prevalence of TB according to the reports by World Health Organization accounting for $46 \%$ of the world's TB cases; Democratic Republic of Congo, South Africa, Kenya, Mozambique, Tanzania, Ethiopia, Nigeria, Zimbabwe and Uganda. The most affected part in Africa is South Africa cutting across East and Central Africa although some of the West African countries also bear the burden of TB while North African Countries are less affected. In Africa, TB burden coincides with HIV/AIDS prevalence and usually serving as a manifestation of the latter. The Millenium Development Goal (MDG) main objective with regard to TB in Africa is to halt and reverse the incidences by 2020 . This has seen various developments and campaigns emerging in the last couple of years.

Kenya being among the highly burdened country in the world contribute to the collective $80 \%$ of the global TB disease burden. The most devastating attribute is that it has a generalized TB prevalence affecting productive age groups (15-44 years old). Males are 1.4 times more likely to have TB than females. The number of TB cases had increased tenfold from 11,625 in 1990 to 116,723 cases in 2007 to 106,083 in 2010. The average annual increase over the past 10 years was $4 \%$ for all forms of TB. In 2012, the total TB cases notified in Kenya were 99,159 cases (MOH and DLTLD, 2012).

The major reason for the increasing burden of TB in Kenya is the concurrent HIV epidemic. People Living with HIV/AIDS (PLWHA) are the major subgroup with increased incidence of tuberculosis. The national average co -infection with HIV was 39\% in 2011 (MOH and DLTLD, 2012). So, TB infection control remains a major programmatic intervention in $\mathrm{TB}$ control especially in this era of HIV epidemic and the increasing prevalence of drug resistant TB. 
Journal of Health, Medicine and Nursing

ISSN 2520-4025 (Online)

Vol.6, Issue 3. No.4, pp 49- 65, 2021

\section{Patient-related factors}

According to self-efficacy concept developed by Albert Bandura in 1977, motivation, thinking, feelings, quality of decision making are influenced by the level of self-efficacy. Peoples selfefficacy believes, determine their level of motivation and how much efforts they will exert in achieving their endeavours (TB treatment completion). Belief in curability, severity of the disease, support from family members and health care professional positively influence TB patient's drug adherence (Gebremariam et al., 2010). Age and gender have been linked to treatment adherence in diferent settings (Hudelson,1996). In a longtitudinal study by Fatigegun et al., (2009), the level of knowledge on the TB disease influenced whether or not patient chooses to complete treatment.

Drug resistant tuberculosis, low BMI, adverse drug events were identified as independent prognostic factors for poor outcomes among TB patients in China (Tang, 2013). In a study done in Zambia in 2008 in patients attending tertiary hospital, patients attitude are significantly associated with TB treatment compliance (Mwemba et al., 2008). Knowledge about curability and importance of adherence are strongly associated with high treatment completion as compared with those lost to follow up according to Tachfouti et al., (2012). More than two fifth of TB clients are lost to follow up within the first two months of treament. This is attributable to ignorance about duration of TB treatment, travelling from treatment sites, improvement in health status and drug adverse effects. Herbal remedies and alcohol was also identified as a factor that hinder TB treatment completion according to Muture et al., ( 2011).

In a systematic review by Barker et al., (2011), they also established that comobidity with diabetes is associated with increased risk of treatment failure and death during treatment and increased chances of relapses later on in life. A prospective cohort study involving pulmonary TB patients with comordity with diabetes (Jepsen et al., 2012) assessed anthropometry and clinical parameters found that diabetes comordity delayed recovery of body mass and haemoglobin which are important functional recovery indicators. In another retrospective study carried in Maryland among tuberculosis patients with co-morbidity (Dooley et al., 2009) established that diabetes is a major risk factor for death among TB patients and there is a trend toward increased time to culture conversion by two months.

In a retrospective case control study in Singapore there was no significant association of defaulting with age, sex, marital or employment status, disease characteristics, or treatmentrelated factors and lack of family support were found to be factors strongly predictive of default, Chee et al., (2000). A recent study to assess the risk of loss to follow among previously treated tuberculosis patients in South Africa, established that previously treatment history, male gender and age group of 19-39 years were independently associated with loss to follow up, Marx et al, (2012)

A cohort study done in Spain among co-infected patients revealed that $11 \%$ of patients were lost to follow up and this was found to be higher among previously treated patients and $6.6 \%$ died, Monge et al., 2014. Unsuccessful treatment was associated with social economic status according to any criteria used, except for the definition of poverty line. Poverty seems to be hampering the achievement of the World Health Organization targeted $90 \%$ cure rate in developing settings (Belo, Luiz, Teixeira, Hanson \& Trajman, 2011). 
Journal of Health, Medicine and Nursing

ISSN 2520-4025 (Online)

Vol.6, Issue 3. No.4, pp 49- 65, 2021

\subsection{RESEARCH METHODOLOGY}

WWw.iprib.org

Study was conducted in Mombasa County which is one of the 47 counties in Kenya with an urban population of $1,063,854$. The study used a cross-sectional research design. The study population was the total number of notified patients with tuberculosis in one quarter in the study area (Mombasa's health care units) and this was found to be 1207 in the year 2017. It was from this population that a systematic random sample size of 292 patients were interviewed. Quantitative data were analyzed using SPSS version 20 Descriptive statistics frequency (\%), mean, and standard deviation were used to express quantitative data. In bivariate analyses, odds ratios (OR) and 95\% confidence intervals (CI) for the association between TB treatment outcome and health related factors, institutional factors and patient related factors was done using logistic regression.

\subsection{PRESENTATION AND INTERPRETATION OF FINDINGS}

\subsection{Demographic characteristics of the respondents and TB-Outcome relationship}

\subsubsection{Gender}

The gender of the participants was almost equally balanced. There were 147 male participants and 118 female participants who accounted for $55.5 \%$ and $44.5 \%$ respectively. However, there was no significant association between gender and TB outcome $\left(\mathrm{X}^{2}=3.174\right.$; df 1 and $p$-value $=$ 0.075).

\subsubsection{Age}

The mean age of the participants was 35 ( \pm SD 9.8) years with a range of 25 to 55 years. Participants who were below 25 years accounted for 5.7\%, while those who 25 to $34 \%$ accounted for a higher percentage of $28.3 \%$. Those who indicated age between 35 to 44 years accounted for the highest percentage of $36.9 \%$ and those who indicated age between 45 to 55 years accounted for a relatively lower percentage (24.2\%) of the participants. Additionally, those who above 55\% accounted for $4.9 \%$. There was no significant association between age and the outcome of the TB treatment $\left(\mathrm{X}^{2}=107.057\right.$; df 4 and $p$-value $\left.=0.055\right)$.

\subsubsection{Marital Status}

Majority of the respondents $192(72.5 \%)$ were married while only 59 (22.3\%) were single and only $14(5.2 \%)$ of the respondents were widowed or divorced. Marital status had a significant association with the outcome of the TB treatment $\left(X^{2}=93.955\right.$; df 2 and $p$-value $\left.=0.001\right)$.

\subsubsection{Religion}

The study sought to identify the association between religion and TB treatment outcome. 164 $(61.9 \%)$ of the respondents were Christians while 101 (38.1\%) of the respondents were Muslims. None of the study participants indicated Hinduism or any other religion. Additionally, there was a significant association between religion and outcome of the TB treatment $\left(X^{2}=14.977\right.$; df 1 and $p$-value $=0.001)$.

\subsubsection{Education}

Majority $174(61.9 \%)$ of the respondents had primary education while those who had secondary education were $54(19.2 \%)$ of the total respondents. Those who attended college were relatively small compared to those who had secondary education with $32(11.4 \%)$ and only $5(1.9 \%)$ 
Journal of Health, Medicine and Nursing

ISSN 2520-4025 (Online)

Vol.6, Issue 3. No.4, pp 49- 65, 2021

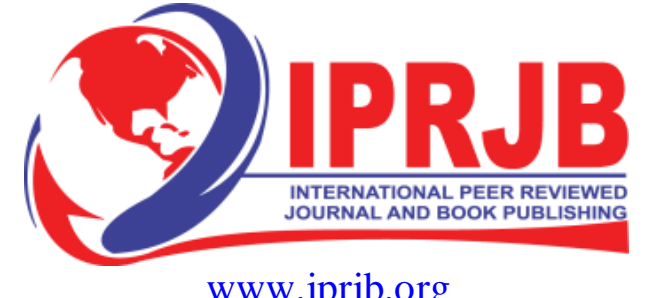

Majority 51.1\% (146) of the respondents indicated that they experienced stigma while a relatively lower number $119(44.9 \%)$ of the respondents indicated that they don't experience stigma. The association between stigma and outcome of the TB treatment was not significant $\left(\mathrm{X}^{2}\right.$ $=5.637 ; \mathrm{df}=4$ and $p$-value $=0.228)$.

\subsection{Odds Ratio for Patient related factors}

Table 4.7 shows that respondent who are treated more than twice are 1.993 times more likely to get cured of TB compared to those who are treated once (OR-1.993, 95\%CI, 0.7 to 5.5). Additionally, respondents who are treated twice are 1.33 times more likely to get cured compared to those who are treated once (OR-1.33, 95\% CI, 0.21 to 3.65).

Patients who smoke cigarette are 0.579 times less likely to get cured as compared to those who don't smoke cigarette (OR-0.579, 95\% CI, 0.26 to 1.28). Additionally, patients who drink alcohol are 0.307 times less likely to get cured of TB as compared to those who don't drink alcohol (OR-0.307, 95\% CI, 0.139 to 0.678). The results also revealed that patients who are educated about health are 1.716 times more likely to cure of TB than patients who are not (OR$1.716,95 \% \mathrm{CI}, 0.35$ to 1.48 ). More so, patients who receive psychosocial support are 4.08 times more likely to get cured than those who don't receive psychosocial support (OR-4.08, 95\% CI, 2.00 to 8.32 ). Finally, it was revealed that patients who has knowledge of illness are more 1.98 times likely to get cured to those who are not knowledgeable (OR-1.98, 95\% CI, 0.17 to 1.47).

Related studies by Dujaili, et al (2010), confirmed a linear relationship between cigarette smoking's with poor treatment outcomes. This study further isolated treatment outcomes with smoking severity. Influence of drinking of alcohol on treatment outcomes was studied by Pelzter and Louwz (2014) in their work that aimed at isolating factors associated with treatment failure and death of patients from TB incidences.

The impact of health education was exhaustively explored and discussed by D Souza (2003) and specifically in the treatment of Pulmonary Tuberculosis. D Souza provides evidence of effect of intensive health education on adherence to treatment which was studied in 60 newly diagnosed pulmonary tuberculosis (TB) patients where their knowledge of TB before and after health education was recorded and their health status during the first three months of the treatment was assessed to measure the effect. His study revealed a statistically significant difference in the total health status scores of patients, after receiving intensive health education between $1^{\text {st }}$ and $30^{\text {th }}$ day, $30^{\text {th }}$ and $60^{\text {th }}$ day, $60^{\text {th }}$ and $90^{\text {th }}$ day, and $1^{\text {st }}$ and $90^{\text {th }}$ day, higher mean post-test knowledge scores, and a highly significant association between sputum conversion and adherence to treatment. Unsuccessful outcome as death, lost-to-follow up, treatment-failure, or not-evaluated and successful outcome as a patient cured or completed-treatment. 
Journal of Health, Medicine and Nursing

ISSN 2520-4025 (Online)

Vol.6, Issue 3. No.4, pp 49- 65, 2021

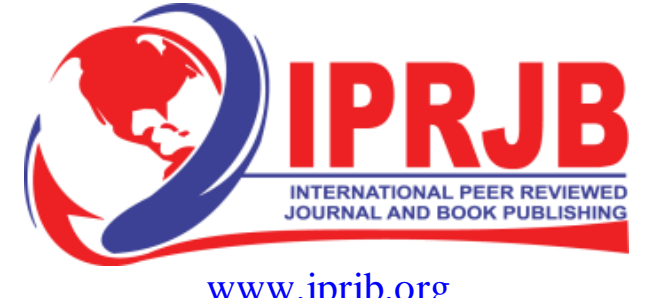

to improve TB burden in the County of Mombasa. This was supported by odds ration results which revealed that patients who smoke cigarette are less likely to get cured as compared to those who don't smoke cigarette (OR $0.579,95 \%$ CI, 0.26 to 1.28). Additionally, patients who drink alcohol are less likely to get cured of TB as compared to those who don't drink alcohol (OR $0.307,95 \% \mathrm{CI}, 0.139$ to 0.678 ). The results also revealed that patients who are educated about health are more likely to cure of TB than patients who are not (OR 1.716, 95\% CI, 0.35 to 1.48). More so, patients who receive psychosocial support are more likely to get cured than those who don't receive psychosocial support (OR 4.08, 95\% CI, 2.00 to 8.32).

\subsection{Conclusion}

Based on the findings of the study, the study therefore, conclude that Patient related factors are significant factors that influence the outcomes of TB among TB patients in Mombasa County. Specifically, among the patient related factors, times treated for TB, cigarette smoking, drink alcohol, health education, psychosocial support, knowledge of illness, side effects and stigma were concluded to be the factors that affect TB outcomes among TB patients. That is, the less the patient smokes cigarette, drinks alcohol the more the patient outcomes are likely to be improved. In addition, patients who are treated more often, and those who are enlightened about their health are more likely to recover from TB. Therefore, it is worth noting that the patient needs to be aware of the patient related factors in order to minimize the risk that are associated with them.

\subsection{Recommendations and Contributions of the Study}

Strengthening TB adherence counselling for patients treated for the first time to improve treatment completion. Counselling service should also be enhanced for patients taking alcohol and those smoking cigarettes as both are associated with less likelihood of completing TB treatment. Implementation of TB work place program through sensitization of company managers on the importance of TB treatment support to reduce chances of loss to follow as sighted by focused group discussions. Patients centred TB services should be provided by all facilities by focusing on the needs of the patients by agreeing on the best time to pick drugs to reduce the risk of loss to follow ups.

\section{REFERENCES}

Arnadottir, T. (2009). Tuberculosis and public health. Policy and principles in tuberculosis control. International union against tuberculosis and lung disease (The Union).

Bandura, A. (1997). Self-efficacy: The exercise of control. New York: W.H. Freeman and Company.

CDC. (2003). Tuberculosis Behavioral and Social science research forum: planting the seeds for future research. Atlanta, GA, CDC.

Chee, C. B. E., Boudville, I. C., Chan, S. P., Zee, Y. K., \& Wang, Y. T. (2000). Patient and disease characteristics, and outcome of treatment defaulters from the Singapore TB control unit-a one-year retrospective survey. The International Journal of Tuberculosis and Lung Disease, 4(6), 496-503.

Dooley, K. E., \& Chaisson, R. E. (2009). Tuberculosis and diabetes mellitus: convergence of two epidemics. The Lancet infectious diseases, 9(12), 737-746. 
